\title{
A influência dos stakeholders na gestão ambiental dos hotéis do Algarve: análise das relações estruturais subjacentes
}

\section{The stakeholders influence in the environmental management of the hotels of Algarve}

\section{Margarida Arraes Viegas}

Universidade do Algarve, Escola Superior de Gestão, Hotelaria e Turismo, Núcleo de Métodos Quantitativos, Campus da Penha, 8005-139 Faro, mmviegas@ualg.pt

\section{Resumo}

No presente trabalho efetua-se uma análise dos stakeholders das unidades hoteleiras algarvias e da influência por eles exercida nas ações de gestão ambiental destes estabelecimentos. Utilizam-se as técnicas estatísticas de Análise de Componentes Principais e de Análise de Equações Estruturais, com recurso aos Mínimos Quadrados Parciais (Partial Least Squares-PLS), método cuja aplicação se pretende exemplificar. Os resultados obtidos permitem diferenciar os stakeholders em função da pressão ambiental que lhes é percecionada e detetar diferentes influências por eles exercidas nas duas dimensões da gestão ambiental empiricamente determinadas.

Palavras chave: Stakeholders, gestão ambiental, hotéis, AEE, PLS.

\begin{abstract}
This article is a study of the environmental stakeholders of the Algarve accommodation sector and their influence on the environmental management of these units. The statistic techniques applied are Principal Components Analysis and Partial Least Squares Structural Equation Modeling, method which application we intend to illustrate. The results indicate two dimensions of environmental management and two types of stakeholders pressure with different impacts on them.
\end{abstract}

Keywords: Stakeholders, environmental management, hotels, SEM, PLS.

\section{Introdução}

A cada vez mais evidente degradação ambiental originada pela atividade empresarial e os sérios problemas ambientais com que a sociedade se tem confrontado nas últimas décadas, suscitam uma tomada de atenção crescente para a responsabilidade das empresas na proteção ambiental, questão que começa a ser debatida com profundidade nas últimas três décadas do séc. XX. Ao longo da década de 90, o novo paradigma da sustentabilidade promove uma abordagem mais ampla aos problemas ambientais e conduz a um aumento da pressão sobre as empresas para que procurem desenvolver métodos de gestão ambiental inovadores.

De acordo com Sharma e Starik (2004), a pressão exercida pelos diversos agentes sociais da organização é uma das principais forças impulsionadoras da gestão ambiental responsável, pelo que a teoria dos stakeholders (ou agentes sociais ou grupos de interesse), pode particularizar-se para explicar o comportamento empresarial face às questões ambientais, como acontece em estudos como os de Alvarez, de Burgos e Céspedes (2001a) e Buysse e Verbek (2003). 0 eixo central sobre o qual assenta esta teoria afirma que a empresa tem, para além da responsabilidade privada para com os seus acionistas, uma responsabilidade pública adicional para com todos os agentes sociais com os quais interatua. Dada a grande diversidade de stakeholders, Fassin (2009) considera não ser possível atender igualmente a todos eles, pelo que a empresa deverá direcionar os seus esforços no sentido de satisfazer as exigências dos agentes sociais realmente importantes para a sua sobrevivência e sucesso. Desta forma, e no contexto da temática ambiental, a empresa orientará as suas ações de gestão ambiental de acordo com as exigências dos grupos de interesse que considera mais importantes.

Dos vários estudos dedicados à análise da atuação ambiental empresarial, na perspetiva da teoria dos stakeholders, grande parte incide sobre empresas do setor industrial. Este setor, tradicionalmente associado a maiores impactos ambientais, tem sido também prioritário em termos de regulamentação ambiental o que, de acordo com Alvarez et al. (2001a), relega para segundo plano a influência de outros agentes sociais. Estas razões atribuem particular interesse à investigação do comportamento ambiental das empresas de serviços, e em especial das empresas de alojamento turístico, dada a menor regulamentação ambiental a que, comparativamente, estão sujeitas.

No caso do Algarve, a análise da temática ambiental no setor turístico assume especial relevância, não só devido à importância económica de que esta atividade se reveste para a região, mas também pelo seu impacto nos recursos naturais, dos quais a sua continuidade depende. 0 presente trabalho pretende contribuir para um melhor conhecimento das especificidades ambientais deste setor, pelo que tem como objetivos identificar quais os principais grupos de interesse das unidades hoteleiras algarvias, como é percecionada a pressão ambiental por eles exercida e qual o efeito desta nas ações de gestão ambiental desenvolvidas por estes estabelecimentos.

\section{Stakeholders e gestão ambiental das unidades hoteleiras}

A atividade hoteleira é constituída por uma grande quantidade de atividades e operações que envolvem o consumo de recursos naturais e que produzem impactos ambientais significativos, pelo que a questão ambiental ganha cada vez mais espaço no sector. As exigências em relação à preocupação e responsabilidade ambientais partem não só da esfera governamental e dos organismos de certificação mas, principalmente, da pressão exercida pelos consumidores. Neste contexto, a gestão ambiental é 
uma ferramenta estratégica que o sector não pode dar-se ao luxo de ignorar.

De acordo com Céspedes e De Burgos (2004), a gestão ambiental para o sector hoteleiro pode ser definida como "um conjunto de ações de natureza diversa, levadas a cabo pelos hotéis, com o objetivo de melhorar a relação do estabelecimento com o ambiente", sendo que uma das classificações mais usuais na literatura divide estas ações em organizacionais (formais ou de software) e operacionais (técnicas, materiais ou de hardware). As atividades organizacionais referem-se à incorporação das questões ambientais dentro dos valores, objetivos e estrutura organizacional da empresa, ao passo que as atividades técnicas dizem respeito à utilização de técnicas ou tecnologias que reduzem o impacto físico resultante da própria atividade empresarial. Para o presente trabalho selecionaram-se 19 ações ambientais, de entre as mais comumente utilizadas na literatura (Aragón-Correa, 1998; Carmona, Céspedes \& de Burgos, 2004; Céspedes \& de Burgos, 2004), solicitando-se aos inquiridos a avaliação do seu nível de implementação através de uma escala tipoLickert de 5 pontos, correspondendo o 1 à implementação nula da medida em causa e 5 à sua total implementação.

Segundo Alvarez et al. (2001a), os grupos de interesse a considerar para os estabelecimentos hoteleiros são: acionistas, administração pública, agências de viagens e operadores turísticos, associação ou cadeia a que pertencem, clientes, concorrentes, diretor do hotel, funcionários, fornecedores e ONG.

Com o objetivo de identificar e classificar por importância os stakeholders de uma organização, diversas propostas surgiram na literatura, sendo a mais utilizada o modelo da Saliência (Stakeholders Salience Modell), desenvolvido por Mitchell, Agle e Wood (1997). De acordo com estes autores, para determinar a saliência/relevância que cada um dos grupos de interesse tem para a empresa, devem considerarse três atributos básicos relativamente a cada um deles: o seu poder (ou capacidade de influenciar a empresa), a sua urgência (ou interesse na resposta da empresa) e a sua legitimidade. Sendo o meio ambiente um bem comum, e de acordo com o sugerido por Alvarez et al. (2001b), assumese neste estudo que existe um interesse legítimo de todos os stakeholders em reivindicar a adoção de medidas de proteção ambiental, restando portanto aferir a sua capacidade de influência sobre o hotel (poder) e a importância atribuída à resposta ambiental dada por este (urgência/interesse). Assim, solicitava-se aos inquiridos por um lado, a valorização de cada um dos grupos de interesse em função da sua capacidade para influenciar o comportamento do hotel e, por outro, a valorização da importância que cada um desses grupos atribuiria às ações ambientais por ele desenvolvidas (ambas as escalas tipoLickert de 5 pontos).

\section{Metodologia e análise empírica}

Para a realização do estudo empírico foi elaborado um questionário na plataforma Surveygyzmo (http://edu.surveygizmo.com/s3/1048658/Gest-o-

Ambiental-no-Setor-Hoteleiro-do-Algarve), dirigido aos responsáveis ambientais/diretores dos 140 hotéis de 2,3,4 e 5 estrelas do Algarve, registados no Registo Nacional de Empreendimentos Turísticos (RNET), disponível no portal do Turismo de Portugal: https://rnt.turismodeportugal.pt/RNET/Registos.Consulta Registo.aspx?Origem=CP\&MostraFiltro=True. 0 período de recolha decorreu de junho a setembro de 2013, obtendo-se um total de 66 questionários validados, a que corresponde uma taxa de resposta de $47 \%$.

Como anteriormente referido, numa primeira fase da análise pretende-se classificar os grupos de interesse do setor hoteleiro, de acordo com a relevância que lhes é atribuída pelas unidades inquiridas. Para a aplicação do modelo da Saliência, e dado que este considera os atributos como binários, considera-se que aqueles que apresentem classificações altas ou muito altas (média $\geq 3.5$ ), podem ser atribuídos aos correspondentes stakeholders, obtendo-se a seguinte classificação (Quadro 1):

\section{Quadro 1 - Stakeholders Salience Model}

\begin{tabular}{|c|c|c|c|c|c|}
\hline \multirow{2}{*}{ Stakeholders } & \multicolumn{2}{|c|}{$\bar{X}$} & \multicolumn{3}{|c|}{ Modelo da Saliência } \\
\hline & Poder & Interesse & Atributos & Saliência & Categoria \\
\hline Diretor & 4.07 & 4.13 & Legitimidade & & \\
\hline Clientes & 3.95 & 3.74 & Poder & Alta & Definitivos \\
\hline Funcionários & 3.54 & 3.50 & Interesse & & \\
\hline Acionistas & 3.93 & 3.32 & Legitimidade e & Moderada & Fxnectantec \\
\hline Agências\&Operadores & 3.56 & 3.43 & Poder & 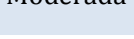 & Барестанте \\
\hline Cadeia & 3.27 & 3.15 & & & \\
\hline Concorrentes & 3.25 & 2.95 & & & \\
\hline Administração Pública & 3.10 & 3.05 & Legitimidade & Baixa & Latentes \\
\hline Fornecedores & 3.03 & 3.03 & & & \\
\hline ONG & 2.67 & 3.07 & & & \\
\hline$\alpha$ de Cronbach & 0.824 & 0.886 & & & \\
\hline
\end{tabular}

Da análise dos valores médios verificamos que a cinco stakeholders é percecionado poder para influenciar a gestão ambiental das unidades, enquanto que a apenas três é reconhecido o interesse nessa mesma gestão. Observa-se também, para a maioria dos casos, um equilíbrio entre os dois atributos; com exceção para os acionistas, concorrentes e clientes que apresentam mais poder do que interesse e para as ONG, nas quais se verifica o inverso (testes $t$ para amostras emparelhadas com $\alpha=5 \%$ e significâncias observadas, respetivamente: $0.001,0.031$, 0.036 e 0.004 ). 
Na segunda parte deste trabalho pretende-se averiguar se a pressão dos agentes sociais influencia a gestão ambiental das unidades hoteleiras inquiridas. Para o efeito procederse-á a uma análise de equações estruturais (AEE), tendo-se previamente submetido as variáveis de interesse a uma análise exploratória de componentes principais (ACP), que se resume nos quadros seguintes. Relativamente à gestão ambiental, a aplicação da ACP indica que as 19 variáveis escolhidas para medir a gestão ambiental dos hotéis podem ser agrupadas em duas dimensões (Quadro 2), resultados coincidentes com os obtidos por diversos autores (AragónCorrea, 1998; Alvarez et al., 2001a; Carmona et al., 2004).

\begin{tabular}{|c|c|c|c|c|c|c|}
\hline \multicolumn{7}{|c|}{ Gestão Ambiental } \\
\hline \multicolumn{3}{|c|}{ Adequabilidade } & \multirow{3}{*}{$\begin{array}{l}\text { Extração de } \\
\text { fatores }\end{array}$} & \multirow{3}{*}{$\begin{array}{c}\text { Rotação de } \\
\text { fatores }\end{array}$} & \multirow{2}{*}{\multicolumn{2}{|c|}{$\begin{array}{c}\text { Critérios } \\
\mathrm{n} \text { o fatores a reter }\end{array}$}} \\
\hline \multicolumn{2}{|c|}{ Teste de Barttlett } & \multirow{2}{*}{ KMO } & & & & \\
\hline X 2 & Sig. & & & & Kaiser & \% variância \\
\hline \multirow{2}{*}{1203.34} & \multirow{2}{*}{0.000} & \multirow{2}{*}{0.899} & \multirow{2}{*}{ Ortogonal } & \multirow{2}{*}{ Varimax } & 12,032 & 46,931 \\
\hline & & & & & 2,154 & 74,662 \\
\hline \multicolumn{4}{|c|}{ “Gestão Ambiental Organizacional” ( $\alpha=0.97)$} & \multicolumn{3}{|c|}{ “Gestão Ambiental Operacional” ( $\alpha=0.89)$} \\
\hline \multicolumn{3}{|c|}{ Variáveis } & Contribuições & \multicolumn{2}{|c|}{ Variáveis } & Contribuições \\
\hline \multicolumn{3}{|c|}{ Responsável ambiental } & ,931 & \multicolumn{2}{|c|}{ Poupança de energia } & ,883 \\
\hline \multicolumn{3}{|c|}{ Iniciativas ambientais } & ,876 & \multicolumn{2}{|c|}{ Poupança de água } & ,873 \\
\hline \multicolumn{3}{|c|}{ Reporte ao exterior } & 872 & \multicolumn{2}{|c|}{ Recolha seletiva } & ,809 \\
\hline \multicolumn{3}{|c|}{ Formação ambiental } & ,834 & \multicolumn{2}{|c|}{ Redução produtos tóxicos } &, 735 \\
\hline \multicolumn{3}{|c|}{ Argumentos ecológicos } & ,832 & \multicolumn{2}{|c|}{ Colaboração hóspedes } & ,684 \\
\hline \multicolumn{3}{|c|}{ Política ambiental } & ,810 & \multicolumn{2}{|c|}{ Produtos ecológicos } & 619 \\
\hline \multicolumn{3}{|c|}{ Quantificação custos/benefícios } &, 796 & & & \\
\hline \multicolumn{3}{|l|}{ SGA } & ,788 & & & \\
\hline \multicolumn{3}{|c|}{ Disposições legais } &, 777 & & & \\
\hline \multicolumn{3}{|c|}{ Interesses stakeholders } & ,758 & & & \\
\hline \multicolumn{3}{|c|}{ Código boas práticas } & ,756 & & & \\
\hline \multicolumn{3}{|c|}{ Ações ambientais LP } &, 751 & & & \\
\hline \multicolumn{3}{|c|}{ Implicação diretiva } &, 660 & & & \\
\hline
\end{tabular}

Fonte: Elaboração própria.

A aplicação da ACP à pressão ambiental dos stakeholders (sendo esta quantificada através da ponderação dos atributos poder e interesse, conforme sugerido por Alvarez et al., 2001), deu origem a duas componentes (Quadro 3), resultado com aderência teórica às categorizações referidas por autores como Clarkson (1995) e Barbieri e Cajazeira (2009).

Quadro 3 - ACP stakeholders

\begin{tabular}{|c|c|c|c|c|c|c|}
\hline \multicolumn{7}{|c|}{ Pressão dos Stakeholders } \\
\hline \multicolumn{3}{|c|}{ Adequabilidade } & \multirow{3}{*}{$\begin{array}{l}\text { Extração de } \\
\text { fatores }\end{array}$} & \multirow{3}{*}{$\begin{array}{l}\text { Rotação de } \\
\text { fatores }\end{array}$} & \multirow{2}{*}{\multicolumn{2}{|c|}{$\begin{array}{c}\text { Critérios } \\
\mathrm{n} \text { - fatores a reter }\end{array}$}} \\
\hline \multicolumn{2}{|c|}{ Teste de Barttlett } & \multirow{2}{*}{ KMO } & & & & \\
\hline $\mathrm{X} 2$ & Sig. & & & & Kaiser & \% variância \\
\hline 258,974 & 0.000 & ,866 & Ortogonal & Varimax & $\begin{array}{l}4,728 \\
1,078\end{array}$ & $\begin{array}{l}41,564 \\
72,573\end{array}$ \\
\hline \multicolumn{4}{|c|}{ "Stakeholders primários" $(\alpha=0.90)$} & \multicolumn{3}{|c|}{ "Stakeholders secundários" $(\alpha=0.79)$} \\
\hline \multicolumn{3}{|c|}{ Variáveis } & Contribuições & \multicolumn{2}{|c|}{ Variáveis } & Contribuições \\
\hline \multicolumn{3}{|l|}{ Fornecedores } & ,859 & \multicolumn{2}{|c|}{ Agências \& Operadores } & ,831 \\
\hline \multicolumn{3}{|l|}{ Funcionários } & ,849 & \multicolumn{2}{|c|}{ Administração Pública } & ,793 \\
\hline \multicolumn{3}{|l|}{ Concorrentes } & ,773 & \multicolumn{2}{|l|}{ Acionistas } & ,767 \\
\hline \multicolumn{3}{|l|}{ Clientes } & ,768 & & & \\
\hline \multicolumn{3}{|l|}{ Diretor } & 694 & & & \\
\hline
\end{tabular}

Não incluídos stakeholders cadeia e ONG (comunalidades inferiores a 0.5).

Fonte: Elaboração própria.

Com base nos resultados anteriores, do modelo estrutural que se apresenta em seguida constarão duas variáveis dependentes (gestão ambiental operacional e organizacional) e duas independentes (stakeholders primários e secundários).

Como é referido por Vieira (2009), a modelização por equações estruturais é uma técnica multivariada que combina análise fatorial confirmatória com modelação econométrica, permitindo testar as estruturas fatoriais dos instrumentos de medida e analisar simultaneamente relações entre múltiplas variáveis latentes (também designadas de constructos, compósitos ou fatores) ou observadas (também designadas de indicadores, itens ou variáveis manifestas). São assim avaliados dois sub- 
modelos: (1) o modelo de medida, que se traduz na relação (cargas fatoriais) entre as variáveis latentes e os seus indicadores e (2) o modelo estrutural que analisa as relações entre os constructos independentes e dependentes do modelo em teste.

Na modelação por equações estruturais, ao contrário das técnicas clássicas que estudam os dados para encontrar uma teoria que os explique, o processo de especificação do modelo é guiado pela combinação de elementos teóricos e de evidências empíricas de pesquisas anteriores, ou seja, como é referido por Marôco (2010, p. 4): "na análise de equações estruturais parte-se de um quadro teórico formulado a priori que será, ou não, confirmado pelos dados recolhidos. A teoria é assim o motor da análise, contrariamente ao paradigma da estatística clássica em que os dados, e não a teoria, estão no centro do processo de análise".

As técnicas estatísticas a utilizar na modelação por equações estruturais podem ser de dois tipos, consoante a matriz de dados utilizada é de covariâncias ou de variâncias. As técnicas baseadas nas covariâncias (Covariance-based Structural Equation Modeling - CB-SEM), são as mais utilizadas e têm por objetivo minimizar a diferença entre a matriz de covariâncias estimada no modelo e a verificada na amostra, ou seja, pretendem explicar as relações existentes entre as variáveis observadas. De acordo com Hair, Black, Babin e Anderson (2010), têm como vantagem a estimação de parâmetros consistentes, não enviesados e eficientes exigindo, contudo, que as variáveis sigam uma distribuição normal e que a amostra seja grande. A técnica baseada nas variâncias (VB-SEM), pretende minimizar a variância residual das variáveis endógenas (tanto latentes como observadas), isto é, tem como objetivo a sua predição. Conhecida como PLS (Partial Least Squares), funciona iterativamente utilizando os Mínimos Quadrados
Ordinários e é apelidada de modelização flexível (soft modeling), por não colocar quaisquer restrições quanto à distribuição das variáveis, à dimensão das amostras e ao nível de medida das variáveis.

De entre as razões, indicadas por autores como Chin e Newsted (1999, apud Moreno, Molina e Moreno 2014, p.114), subjacentes à escolha da técnica PLS, no caso do presente estudo podem citar-se as seguintes: dados provenientes de distribuições não normais, design nãoexperimental da investigação, natureza exploratória do estudo e reduzida dimensão da amostra. De acordo com Chin (1998), a dimensão de amostra requerida para aplicação da técnica PLS será de dez vezes a maior das seguintes possibilidades:

- Número de indicadores do constructo formativo mais complexo;

- Maior número de constructos antecedentes dos constructos endógenos.

Como se verá seguidamente, o modelo formulado não apresenta constructos formativos e nenhum constructo endógeno tem mais do que dois antecedentes, pelo que a dimensão mínima da amostra seria de 20 elementos, inferior aos 64 casos incluídos na subsequente modelização por equações estruturais. No entanto, como referido por Roldán e Sánchez (2012), a estimação da dimensão da amostra para um modelo PLS requer a análise da potência estatística (statistical power analysis), baseada na maior equação de regressão presente no modelo. Uma vez que não possuímos nenhuma regressão com mais do que duas variáveis exógenas e assumindo, de acordo com o proposto por Cohen (1988), um efeito de magnitude média, uma potência de 0.8 e um nível de significância de 0.05 , a amostra deverá possuir 63 ou 66 elementos, conforme critérios constantes do quadro 4 :

Quadro 4 - Dimensão amostra

\begin{tabular}{|c|c|c|c|c|c|c|}
\hline \multirow{2}{*}{$\begin{array}{c}\text { Number of } \\
\text { predictors }\end{array}$} & \multicolumn{4}{|c|}{ Sample sizes based on power analysis } & \multicolumn{3}{c|}{ Sample sizes based on two-step rule-of-thumb } \\
\cline { 2 - 6 } & \multicolumn{3}{|c|}{ Effect size } & \multicolumn{3}{|c|}{ Effect size } \\
\hline 2 & Small & Medium & Large & Small & Medium & Large \\
\hline
\end{tabular}

Fonte: Green (1991, p.503).

0 modelo proposto é esquematizado na Figura 1:

\section{Figura 1 - Representação gráfica do modelo proposto}

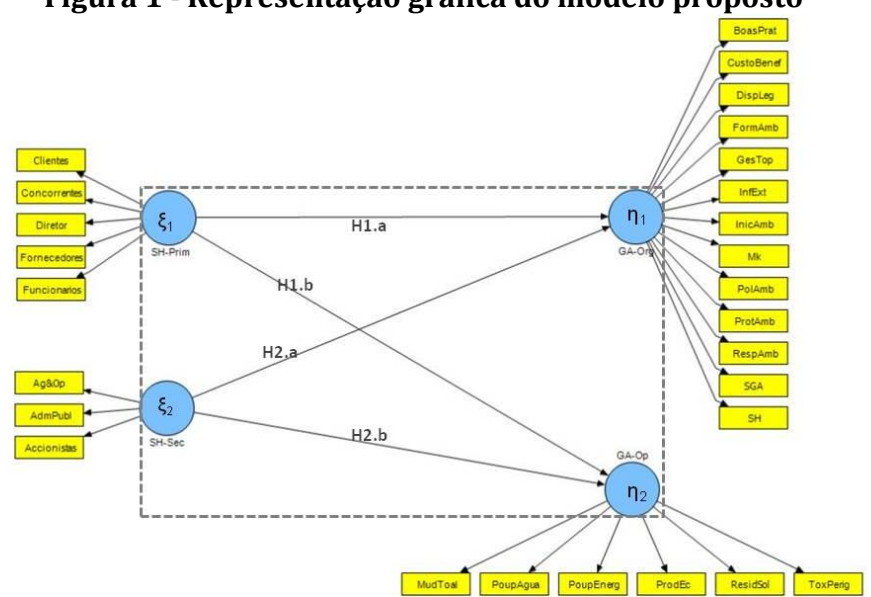

De acordo com a notação comummente utilizada nos modelos

de equações estruturais e apresentada no Quadro 5, 
Quadro 5 - Notação

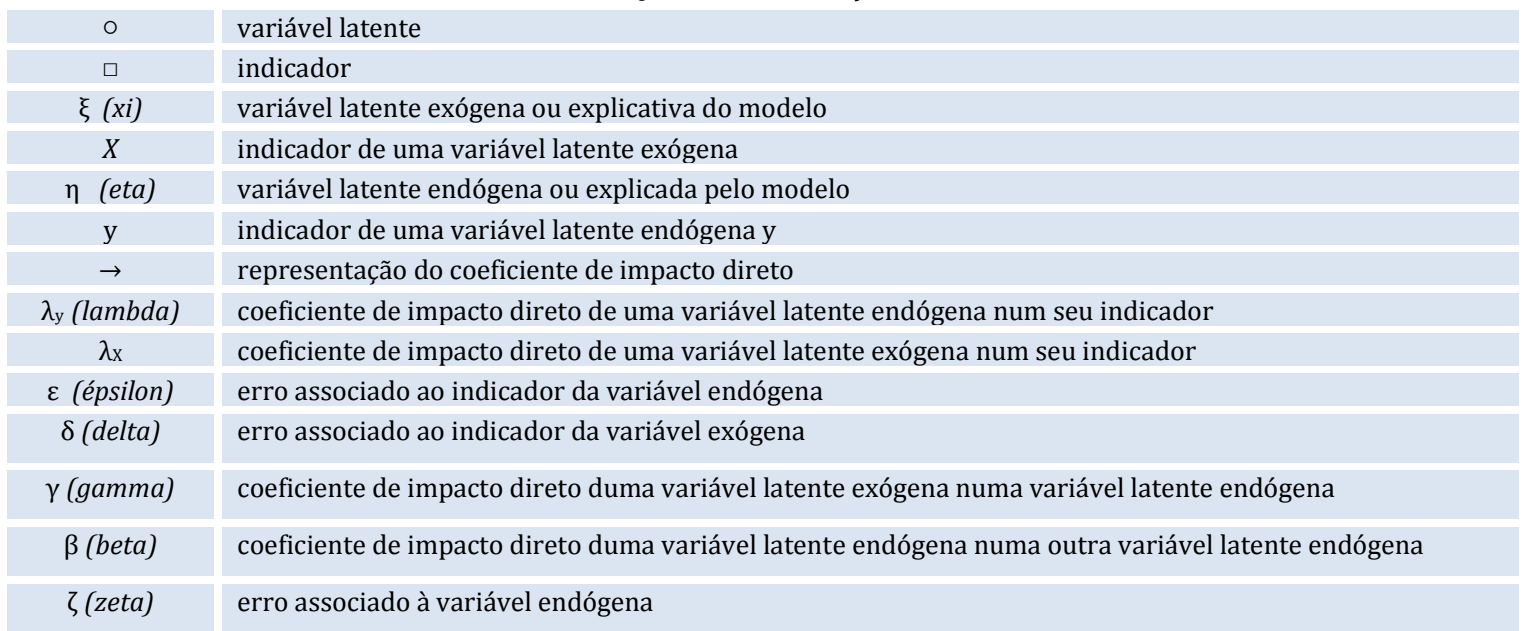

Fonte: Elaboração própria.

as equações do modelo em análise podem escrever-se:

(i) Modelo de medida

$$
\begin{array}{rlrl}
x_{i}=\lambda_{X_{1 i}} \xi_{1}+\delta_{i} & \mathrm{i}=1,2,3,4,5 \\
x_{i}=\lambda_{X_{2 i}} \xi_{2}+\delta_{i} & \mathrm{i}=6,7,8 \\
y_{i}=\lambda_{y_{1 i}} \eta_{1}+\varepsilon_{i} & \mathrm{i}=1,2,3,4,5,6 \\
y_{i}=\lambda_{y_{2 i}} \eta_{2}+\varepsilon_{i} & \mathrm{i}=7, \ldots \ldots \ldots \ldots 1
\end{array}
$$

(ii) Modelo estrutural

$\eta_{1}=\gamma_{11} \xi_{1}+\gamma_{12} \xi_{2}+\zeta_{1}$

$\eta_{2}=\gamma_{21} \xi_{1}+\gamma_{22} \xi_{2}+\zeta_{2}$

Sendo as hipóteses em estudo:

H1: A pressão ambiental dos stakeholders primários influencia de forma positiva:

a) a adoção de medidas organizacionais de gestão ambiental pelos hotéis;

b) a adoção de medidas operacionais de gestão ambiental pelos hotéis;

H2: A pressão ambiental dos stakeholders secundários influencia de forma positiva:

a) a adoção de medidas organizacionais de gestão ambiental pelos hotéis: b) a adoção de medidas operacionais de gestão ambiental pelos hotéis.

A estimação do modelo será efetuada com recurso à técnica PLS, utilizando-se o software SmartPLS (versões 2.0.M3 e 3.0).

\section{Avaliação do modelo de medida}

A avaliação do modelo de medida incide nos atributos validez (variável observada mede realmente o que se deseja medir?) e fiabilidade (fá-lo duma forma estável e consistente?). Analisa-se assim a fiabilidade individual dos itens, a consistência interna ou fiabilidade dos constructos, a validez convergente e a discriminante.

Fiabilidade individual: Num modelo PLS a fiabilidade individual dos itens é avaliada examinando as suas cargas, ou correlações, com os respetivos constructos. A regra empírica mais difundida é a proposta por Carmines e Zeller (1979), de acordo com a qual um indicador deve ser aceite como integrante dum constructo se apresentar uma carga $\geq$ 0.707, o que significa que pelo menos $50 \%$ da variância da variável observada é partilhada pelo constructo. Como se

\begin{tabular}{|c|c|c|c|c|}
\hline Constructos & Indicadores & Abrev. Indicadores & $\begin{array}{c}\text { Cargas } \\
(\lambda)\end{array}$ & t Student \\
\hline \multirow{5}{*}{$\begin{array}{l}\text { Stakeholders } \\
\text { Primários } \\
\text { (SH-Prim) }\end{array}$} & Pressão dos Clientes & Clientes & 0.811 & 14.291 \\
\hline & Pressão dos Concorrentes & Concorrentes & 0.764 & 14.400 \\
\hline & Pressão do Diretor & Diretor & 0.745 & 7.719 \\
\hline & Pressão dos Fornecedores & Fornecedores & 0.730 & 7.599 \\
\hline & Pressão dos Funcionários & Funcionários & 0.827 & 25.246 \\
\hline \multirow{3}{*}{$\begin{array}{c}\text { Stakeholders } \\
\text { Secundários } \\
\text { (SH-Sec) }\end{array}$} & $\begin{array}{l}\text { Pressão das Agências e Operadores } \\
\text { Turísticos }\end{array}$ & Ag\&Op & 0.730 & 2.929 \\
\hline & Pressão da Administração Pública & AdmPubl & 0.827 & 5.554 \\
\hline & Pressão dos Acionistas & Acionistas & 0.850 & 5.061 \\
\hline \multirow{6}{*}{$\begin{array}{c}\text { Gestão Ambiental } \\
\text { Operacional } \\
\text { (GA-Op) }\end{array}$} & Compra produtos ecológicos & ProdEc & 0.809 & 17.273 \\
\hline & Colaboração ambiental clientes & MudToal & 0.736 & 7.499 \\
\hline & Produtos tóxicos e perigosos & ToxPerig & 0.820 & 9.567 \\
\hline & Recolha resíduos sólidos & ResidSol & 0.822 & 13.705 \\
\hline & Poupança de água & PoupAgua & 0.831 & 9.302 \\
\hline & Poupança de energia & PoupEnerg & 0.851 & 12.530 \\
\hline
\end{tabular}
pode ver no quadro 6, todos os indicadores cumprem esta regra. Verifica-se também que todos os itens são significativos a $5 \%$ pois a estatística t apresenta, para todos os casos, valores superiores a 1.96 .

\section{Quadro 6 - Fiabilidade individual}




\begin{tabular}{|c|c|c|c|c|}
\hline Constructos & Indicadores & Abrev. Indicadores & $\begin{array}{c}\text { Cargas } \\
(\lambda)\end{array}$ & t Student \\
\hline \multirow{13}{*}{$\begin{array}{l}\text { Gestão Ambiental } \\
\text { Organizacional } \\
\text { (GA-Org) }\end{array}$} & Política ambiental & PolAmb & 0.827 & 43.426 \\
\hline & Responsável ambiental & RespAmb & 0.834 & 18.983 \\
\hline & Interesses ambientais dos stakeholders & SH & 0.890 & 35.999 \\
\hline & Gestão de topo & GesTop & 0.809 & 17.296 \\
\hline & Formação ambiental & FormAmb & 0.840 & 19.947 \\
\hline & Iniciativas ambientais & InicAmb & 0.871 & 24.603 \\
\hline & Quantificação custos/benefícios & CustoBenef & 0.858 & 19.051 \\
\hline & Reporte ao exterior & InfExt & 0.898 & 28.757 \\
\hline & Argumentos ecológicos & Mk & 0.843 & 49.117 \\
\hline & Disposições legais & DispLeg & 0.878 & 29.088 \\
\hline & SGA & SGA & 0.861 & 18.390 \\
\hline & Código boas práticas & BoasPrat & 0.899 & 29.556 \\
\hline & Medidas proteção ambiental & ProtAmb & 0.893 & 17.293 \\
\hline
\end{tabular}

Fonte: Elaboração própria.

Fiabilidade composta: A avaliação da fiabilidade dos constructos permite comprovar a consistência interna de todos os indicadores na medição do conceito, ou seja, o rigor com que os indicadores medem a mesma variável latente. Esta avaliação processa-se através do alfa de Cronbach $(\alpha)$ e do rho de Dillon-Goldstein $\left(\rho_{c}\right)$, devendo este último, de acordo com Nunnally (1978), assumir valores idênticos ao alfa de Cronbach $(\geq 0.7)$. Como se pode verificar no quadro 7 , todos os constructos apresentam bons níveis de fiabilidade composta em ambos os indicadores.

Validez convergente: Se todos os itens utilizados para medir um constructo, o medem realmente, o seu ajustamento será significativo e estarão altamente correlacionados. De acordo com Fornell e Lacker (1981), esta validez é estabelecida se a variância extraída média (Average Variance Extracted - AVE), apresentar valores superiores a 0.5 , o que significa que mais de $50 \%$ da variância do constructo é devida aos seus indicadores, condição que se verifica para todos os constructos do modelo proposto (quadro 7).

Validez discriminante: Indica-nos em que medida um dado constructo é diferente dos restantes. De acordo com Fornell e Lacker (1981), a validez discriminante de um constructo é confirmada se a quantidade de variância que este partilha com os seus indicadores (AVE), for superior à variância partilhada com os restantes constructos do modelo (correlações ao quadrado entre o constructo e cada um dos restantes). Para simplificar o procedimento de cálculo é comum realizar o procedimento inverso, ou seja, comparar a raiz quadrada de AVE com os valores das correlações entre os constructos. Como se pode ver, no quadro abaixo, todas as raízes quadradas de AVE (valores a negrito na diagonal principal) são superiores às correlações, pelo que podemos afirmar que todos os constructos utilizados possuem validez discriminante (Quadro 7).

Quadro 7 - Fiabilidade composta, validez convergente e discriminante

\begin{tabular}{|c|c|c|c|c|c|c|c|}
\hline Constructos & $\boldsymbol{\alpha}$ & $\boldsymbol{\rho} \mathbf{c}$ & $\mathbf{A V E}$ & $\mathbf{S H - P r i m}$ & $\mathbf{S H - S e c}$ & $\mathbf{G A - O p}$ & GA-Org \\
\hline SH-Prim & 0,8469 & 0,8905 & 0,6204 & $\mathbf{0 . 7 8 7 6}$ & & & \\
\hline SH-Sec & 0,7299 & 0,8452 & 0,6463 & 0,4865 & $\mathbf{0 . 8 0 3 9}$ & \\
\hline GA-Op & 0,8983 & 0,9207 & 0,6598 & 0,4609 & 0,2936 & $\mathbf{0 . 8 1 2 3}$ & \\
\hline GA-Org & 0,9721 & 0,9749 & 0,7493 & 0,5295 & 0,1967 & 0,6686 & $\mathbf{0 . 8 6 5 6}$ \\
\hline
\end{tabular}

Fonte: Elaboração própria.

Uma vez confirmados os critérios de fiabilidade e validez, podemos considerar que temos um modelo de medida satisfatório, pelo que passaremos de seguida à avaliação do modelo estrutural.

\section{Avaliação do Modelo Estrutural}

A avaliação do modelo estrutural deve começar pela análise dos coeficientes de impacto (path coefficients) que, similarmente aos coeficientes de regressão tradicionais, medem o impacto duma variação unitária na variável latente exógena sobre a variável latente endógena. No quadro abaixo apresentam-se os coeficientes de impacto obtidos bem como a sua significância estatística, sendo os valores t de Student obtidos através da técnica de avaliação cruzada designada como Bootstrapping (técnica não paramétrica que estima a precisão e estabilidade dos parâmetros PLS, reestimando o modelo em várias amostras, $\mathrm{m}=1000$, com reposição e com dimensão igual à amostra inicial), que permitiu também o cálculo de intervalos de confiança através dos percentis pois, como é referido por Henseler, Ringle e Sinkovics (2009, p. 306): "se o intervalo de confiança para um parâmetro estimado não inclui o zero, a hipótese de que esse parâmetro seja nulo é rejeitada". É ainda estabelecida a relação entre estes resultados e as hipóteses inicialmente formuladas (Quadro 8). 
Quadro 8 - Análise coeficientes de impacto

\begin{tabular}{|c|c|c|c|c|}
\hline $\begin{array}{c}\text { Relação estrutural com } \\
\text { Gestão Ambiental Organizacional }\end{array}$ & Coef. impacto & $\begin{array}{c}\text { Valor } \\
t\end{array}$ & $\begin{array}{l}\text { Percentil } 95 \% \\
\text { IC }\end{array}$ & Resultado \\
\hline Stakeholders Primários & $\gamma_{11}=0.568$ & 6.477 & $0.089 ; 0.691$ & H1.a) Confirmada** \\
\hline Stakeholders Secundários & $\gamma_{12}=-0.080$ & 0.643 & $-0.256 ; 0.500$ & H2.a) N confirmada \\
\hline \multicolumn{5}{|l|}{$\begin{array}{c}\text { Relação estrutural com } \\
\text { Gestão Ambiental Operacional }\end{array}$} \\
\hline Stakeholders Primários & $\gamma_{21}=0.417$ & 2.673 & $0.367 ; 0.747$ & H1.b) Confirmada*** \\
\hline Stakeholders Secundários & $\gamma_{22}=0.091$ & 0.502 & $-0.317 ; 0.208$ & H2.b) N confirmada \\
\hline
\end{tabular}

${ }^{* * *} \mathrm{p}<0.001,{ }^{* *} \mathrm{p}<0.01$, : (teste $\mathrm{t}_{(999)}$, unilateral). $\mathrm{t}_{(0.001,999)}=3.11, \mathrm{t}_{(0.01,999)}=2.33$

Fonte: Elaboração própria.

Como se pode verificar, existem duas hipóteses não confirmadas, o que nos leva a concluir que a pressão dos stakeholders secundários não tem influência nas ações de gestão ambiental, tanto de caráter organizacional como operacional.

As equações do modelo estrutural podem agora escreverse:

GAOrg $=0.568$ SHPrim -0.080 SHSec $+\zeta_{1}$ GAOp $=0.417$ SHPrim +0.091 SHSec $+\zeta_{2}$
Uma vez calculados os coeficientes de impacto, é agora possível calcular a variância explicada das variáveis endógenas que, de acordo com Falk e Miller (1992), se obtém multiplicando o coeficiente de impacto entre dois constructos pelo valor da correlação entre eles. Segundo os mesmos autores, cada variável preditora deverá explicar, pelo menos, $1.5 \%$ da variância da variável latente endógena, o que se verifica em todas as situações, apesar da percentagem de variância do constructo gestão ambiental organizacional, explicada pelo constructo Stakeholders secundários, se encontrar muito próximo do valor limite (Quadro 9).

Quadro 9 - Variância explicada das variáveis endógenas

\begin{tabular}{|l|c|c|c|}
\hline \multicolumn{1}{|c|}{ Relações modelo estrutural } & Coef. impacto & Correlações & Variância explicada \\
\hline SH-Prim -> GA-Op & 0.417 & 0,4609 & $19.22 \%$ \\
\hline SH-Sec -> GA-Op & 0.091 & 0,2936 & $2.67 \%$ \\
\hline SH-Prim -> GA-Org & 0.568 & 0,5295 & $30.08 \%$ \\
\hline SH-Sec -> GA-Org & -0.080 & 0,1967 & $1.57 \%$ \\
\hline
\end{tabular}

Fonte: Elaboração própria.

À semelhança da análise anterior, verifica-se que os stakeholders secundários têm baixo poder contributivo para a previsão de qualquer dos tipos de ações de gestão ambiental.

Por sua vez, o valor de $\mathrm{R}^{2}$ para os constructos dependentes indica, à semelhança do que acontece numa análise de regressão múltipla, a quantidade de variância do constructo que é explicada pelo modelo. Segundo Falk e Miller (1992), este valor deverá ser, pelo menos, de 0.1 pois valores inferiores, ainda que estatisticamente significativos, proporcionam muito pouca informação e as correspondentes relações possuem um nível preditivo muito reduzido. Como podemos comprovar no quadro anterior, o modelo apresenta um adequado poder preditivo.

Para além da análise do nível de significância das relações entre os constructos e das respetivas variâncias explicadas, a qualidade do modelo estrutural pode ainda ser avaliada através da estatística $Q^{2}$ de Stone-Geisser. Este teste indica em que medida os valores observados são reproduzidos pelo modelo e pelos parâmetros estimados e, de acordo com Chin (1998), deve ser usado para avaliar a relevância preditiva do modelo estrutural, sendo esta confirmada para valores de $\mathrm{Q}^{2}$ superiores a 0 . Conforme se pode constatar (quadro 10), ambos os constructos endógenos cumprem com esta condição, pelo que podemos concluir que o modelo possui relevância preditiva.

\section{Quadro 10 - Relevância preditiva}

\begin{tabular}{|l|l|}
\hline $\begin{array}{c}\text { Constructos } \\
\text { endógenos }\end{array}$ & $\mathbf{Q}^{2}$ \\
\hline GA-Op & 0.1104 \\
\hline GA-Org & 0.2028 \\
\hline
\end{tabular}

Fonte: Elaboração própria.

Tenenhaus, Vinzi, Chatellin e Lauro (2005), propõem, para validação global do modelo, um índice (Goodness-of-Fit GoF), que representa o compromisso entre a qualidade de ambos os sub-modelos e que resulta da média geométrica da comunalidade média e do $\mathrm{R}^{2}$ médio. Assim:

$$
G o F=\sqrt{\overline{A V E} \times \overline{R^{2}}}=\sqrt{0.705 \times 0.252}=0.422
$$

Conforme referido por Akter, D'Ambra e Ray (2011), o valor do critério GoF classifica-se em reduzido (GoF=0.1), moderado $(\mathrm{GoF}=0.25)$ e elevado $(\mathrm{GoF}=0.36)$, pelo que o valor obtido nos permite considerar o modelo como globalmente válido.

A utilização deste critério tem sido questionada por autores como Hair, Hult, Ringle e Sarstedt (2014), que o consideram incapaz de distinguir entre modelos válidos e inválidos. Henseler, Ringle e Sarstedt (2015), propõem, para avaliação global do modelo, a utilização do índice SRMR (Standardized Root Mean Square Residual), que avalia a magnitude média das discrepâncias entre as correlações observadas e as 
esperadas. Como é destacado por Sarstedt, Ringle, Henseler e Hair (2014), o SRMR não possui ainda limiares perfeitamente estabelecidos mas, de acordo com Ringle, Wende e Becker (2015b), pode considerar-se que valores abaixo de 0.1 são indicativos de um ajustamento adequado. É o caso do presente modelo que apresenta um SRMR = 0.094 .
Uma vez que não foram confirmadas as hipóteses relativas à influência dos stakeholders secundários sobre as ações de gestão ambiental das unidades hoteleiras, procedeu-se à reestimação do modelo, agora sem incluir este tipo de agentes sociais, o que significa estarem apenas em análise as hipóteses H1.a e H1.b (Figura 2).

\section{Figura 2: Resultados do modelo final}

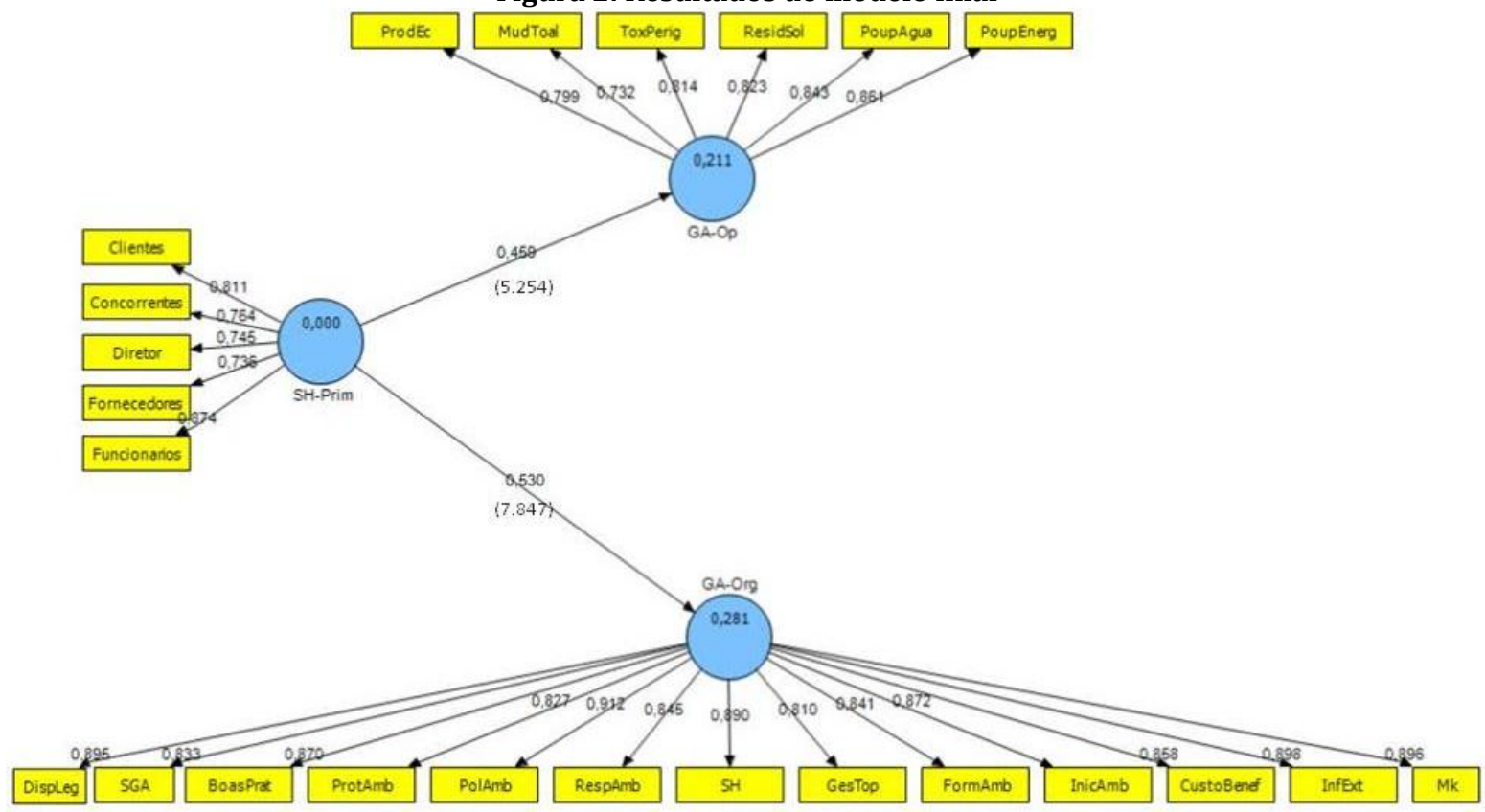

Conforme quadro 11, verifica-se que o modelo de medida cumpre todos os critérios de fiabilidade e validez:

Quadro 11 - Fiabilidade composta, validez convergente e discriminante

\begin{tabular}{|l|c|c|c|c|c|c|}
\hline \multicolumn{1}{|c|}{ Constructos } & $\boldsymbol{\alpha}$ & $\boldsymbol{\rho} \mathbf{c}$ & AVE & SH-Prim & GA-Op & GA-Org \\
\hline SH-Prim & 0,8469 & 0,8905 & 0,6204 & $\mathbf{0 . 7 8 7 7}$ & & \\
\hline GA-Op & 0,8983 & 0,9211 & 0,6611 & 0,4589 & $\mathbf{0 . 8 1 3 1}$ & \\
\hline GA-Org & 0,9721 & 0,9749 & 0,7493 & 0,5297 & 0,6660 & $\mathbf{0 . 8 6 5 6}$ \\
\hline
\end{tabular}

Fonte: Elaboração própria.

Da análise dos coeficientes de impacto e da sua significância estatística (quadro 12), concluímos pela aceitação de ambas as hipóteses, ou seja, confirma-se a influência dos stakeholders primários sobre os dois tipos de ações de gestão ambiental.

Quadro 12: Coeficientes de impacto

\begin{tabular}{|c|c|c|c|c|}
\hline $\begin{array}{c}\text { Relação estrutural com } \\
\text { Gestão Ambiental Operacional }\end{array}$ & Coef. impacto & $\begin{array}{c}\text { Valor } \\
\text { t }\end{array}$ & $\begin{array}{c}\text { Percentil 95\% } \\
\text { IC }\end{array}$ & Resultado \\
\hline Stakeholders Primários & 0.459 & 5.254 & $0.320 ; 0.661$ & H1.a Confirmada* \\
\hline $\begin{array}{c}\text { Relação estrutural com } \\
\text { Gestão Ambiental Organizacional }\end{array}$ & & & \\
\hline Stakeholders Primários & 0.530 & 7.847 & $0.407 ; 0,679$ & H1.b Confirmada* \\
\hline
\end{tabular}
${ }^{*} \mathrm{p}<0.001$

Fonte: Elaboração própria.

Como se pode ver no quadro13, o modelo explica $21.1 \%$ da variação na gestão ambiental operacional e $28.1 \%$ na organizacional, com um poder explicativo médio de $24.6 \%$, valor superior ao mínimo de 10\% recomendado por Falk e Miller (1992).

Quadro 13 - Variância explicada das variáveis latentes

\begin{tabular}{|l|c|c|c|c|}
\hline \multicolumn{2}{|c|}{ Relações modelo estrutural } & Coef. impacto & Correlações & Variância explicada \\
\hline SH-Prim -> GA-Op & 0.459 & 0,4589 & 0.2106 & $21.10 \%$ \\
\hline SH-Prim -> GA-Org & 0.530 & 0,5297 & 0.2807 & $28.10 \%$ \\
\hline
\end{tabular}

Fonte: Elaboração própria. 
Podemos ainda concluir, de acordo com os valores de $\mathrm{Q}^{2}$, GoF e SRMR (quadro 14), que o modelo apresenta relevância preditiva e é globalmente adequado.

\begin{tabular}{|c|c|}
\hline $\begin{array}{c}\text { Quadro 14: Relevância preditiva } \\
\text { Constructos } \\
\text { endógenos }\end{array}$ & $\mathbf{Q}^{2}$ \\
\hline GA-Op & 0,1097 \\
\hline GA-Org & 0,1998 \\
\hline GoF $=0.416$ & \\
\hline SRMR $=0.098$ \\
\hline
\end{tabular}

Fonte: Elaboração própria.

\section{Conclusões}

Este estudo pretende contribuir para a investigação na área da gestão ambiental e para a generalização da aplicação da teoria dos stakeholders nesta temática.

De acordo com a aplicação do modelo de Mitchell et al. (1997), às unidades hoteleiras inquiridas, verifica-se que os agentes sociais podem ser classificados em três níveis de importância: num primeiro, posicionam-se diretores, clientes e funcionários a quem, para além da legitimidade, é reconhecido poder para influenciar a atuação ambiental do hotel e interesse nessa mesma atuação, num segundo nível, classificam-se os acionistas e as agências e operadores turísticos a quem se reconhece poder, mas não interesse, e por último, apenas com legitimidade, todos os restantes.

Constata-se igualmente que os hotéis inquiridos diferenciam os agentes sociais de acordo com a pressão ambiental que lhes percecionam, identificando-se através da ACP duas componentes distintas. Esta diferenciação está em linha com o referido por grande número de autores que, apesar da utilização de diferentes critérios de categorização, consideram a necessidade de considerar duas dimensões relativamente aos grupos de interesse (Freeman, 1984; Clarkson, 1995; Atkinson, Waterhouse e Wells, 1997, Barbieri \& Cajazeira, 2009). No presente trabalho, a primeira dimensão engloba diretores, funcionários, clientes, concorrentes e fornecedores, pelo que se apelidou de stakeholders primários. A segunda, onde se incluem acionistas, administração pública e agências e operadores turísticos, foi designada como stakeholders secundários.

Da mesma forma, confirmou-se o caráter bidimensional da gestão ambiental (operacional e organizacional), por diversas vezes referido na literatura (Aragón, 1998; Alvarez et al., 2001a; Carmona et al., 2004).

A incorporação destes resultados no modelo estrutural, definido com o objetivo de determinar a influência da pressão ambiental dos agentes sociais nas ações de gestão ambiental, permitiu concluir que esta é nula para o caso dos stakeholders secundários, o mesmo não se verificando para os primários cujo impacto, direto e positivo, é empiricamente constatado. A sua influência é significativa em ambas as dimensões da gestão ambiental, embora mais expressiva nas ações de caráter organizacional, o que nos permite concluir que as medidas ambientais adotadas pelos hotéis inquiridos dependem da pressão que percecionam aos seus funcionários, clientes, diretores, concorrentes e fornecedores. Incluindo-se neste grupo os agentes sociais classificados como definitivos pelo modelo da Saliência, podemos ainda concluir que os hotéis inquiridos estabelecem uma relação ativa com os seus stakeholders ambientais mais importantes, tal como preconizado pelo modelo. É de notar a não inclusão dos acionistas nesta categoria, resultado semelhante ao encontrado por Alvarez et al. (2001b) e que poderá significar que as questões ambientais não constam das suas prioridades.

Os resultados obtidos confirmam a existência de conexão entre a perceção da pressão de determinados agentes sociais e a aplicação de medidas de gestão ambiental, o que evidencia a utilidade da teoria dos stakeholders na explicação do comportamento ambiental das unidades hoteleiras. Esta conclusão é particularmente importante no contexto de um setor não industrial e menos sujeito à pressão da legislação ambiental. Segundo Carmona et al. (2004), este tipo de pressão é benéfico para as empresas, levando-as a adotar atitudes ambientais mais proactivas. Em setores menos regulamentados esse efeito será menor, traduzindo-se num maior protagonismo dos restantes stakeholders e revestindo-se, por isso, da maior importância o conhecimento dos mecanismos de resposta das empresas à sua influência. 0 presente trabalho contribui para esse conhecimento, na medida em que se concluiu que os hotéis inquiridos não percecionam na administração pública pressão significativa para a implementação de medidas ambientais, sendo sim os seus clientes, funcionários, diretores, concorrentes e fornecedores a influenciar a sua conduta ambiental.

Uma das limitações deste estudo decorre do próprio modelo estrutural que, apesar de apresentar resultados satisfatórios, pode ser melhorado com a inclusão de variáveis exógenas adicionais. A autosseleção das unidades respondentes e a formulação das questões em função das perceções diretivas podem ainda conduzir a enviesamentos, não permitindo a generalização dos resultados.

\section{Referências}

Akter, S.; D'Ambra, J. \& Ray, P. (2011). An Evaluation of PLS based Complex Models: The Roles of Power Analysis, Predictive Relevance and GoF Index. Proceedings of the 17th Americas Conference on Information Systems (AMCIS2011). (pp. 1-7). Detroit. Alvarez, M., de Burgos, J. \& Céspedes, J. (2001a). Grupos de interes y estrategias medioambientales de los hoteles españoles. Tribuna de Economia, 790 (2), 163-181.

Alvarez, M., de Burgos, J. \& Céspedes, J. (2001b). Un análisis exploratório de las estrategias medioambientales y el contexto organizativo de los hoteles españoles. Cuadernos de Economia y Dirección de la Empresa, 8(1), 5-32.

Aragón-Correa, J. (1998). Strategic proactivity and firm approach to the natural environment. Academic of Management Journal, 41(5), 558-567.

Atkinson, A., Waterhouse, J., Wells, R. (1997). A stakeholder approach to strategic performance measurement, Sloan Management Review, 38(3), 25-37.

Barbieri, J. \& Cajazeira, J. (2009). Responsabilidade social empresarial e empresa sustentável: da teoria à prática. São Paulo: Saraiva.

Buysse, K. \& Verbeke, A. (2003). Proactive environmental strategies: a stakeholder management perspective. Strategic Management Journal, 24(5), 453-470.

Carmines, E. \& Zeller, R. (1979). Reliability and validity assessment. Beverly Hills, CA: Sage. 
Carmona, E., Céspedes, J. \& de Burgos, J. (2004). Environmental strategies in Spanish Hotels: contextual factors and performance. The Service Industries Journal, 24(3), 101-130.

Céspedes, J. \& De Burgos, J. (2004), Un análisis de las dimensiones de la gestión ambiental en los servicios hoteleros. Dirección y Organización, 30(1), 5-15.

Chin, W. (1998). The partial least square approach to structural equation modelling. In G.A. Marcoulides (ed.), Modern methods for business research (pp. 295-336). London: Lawrence Erlbaum Associates.

Clarkson, M. (1995). A stakeholder Framework for analyzing and evaluating corporate social performance. Academy of Management Review, 20(1), 92-117.

Cohen, J. (1988). Statistical power analysis for the behavioral sciences. ( $2{ }^{\mathrm{a}}$ ed). New Jersey: Hillsdale.

Falk, R. \& Miller, N. (1992). A Primer for Soft Modelling. Ohio: Akron. Fassin, Y. (2009). The Stakeholder Model Refined. Journal of Business Ethics, 84(1), 113-135.

Fornell, C. \& Lacker, D. (1981). Evaluating structural equation models with unobservable variables and measurement erros: algebra and statistics. Journal of Marketing Research, 28(2), 39-50.

Freeman, R. (1984). Strategic Management: a stakeholders approach. Boston: Pitman.

Green, S. (1991). How many subjects does it take to do a regression analysis. Multivariate Behavioral Research, 26(3), 499-510.

Hair, J., Black, W., Babin, B. \& Anderson, R. (2010). Multivariate data analysis. (7를. $)$, New Jersey: Prentice-Hall.

Hair, J.; Hult, T.; Ringle, C. \& Sarstedt, M. (2014). A primer on partial least squares structural equation modeling (PLS-SEM). Los Angeles: Sage.

Henseler, J., Ringle, C. \& Sinkovics, R. (2009). The use of partial least squares path modeling in international marketing. In R. Sinkovics \& P. Ghauri (eds.). Advances in international marketing (pp. 277-319). Bingley: Emerald Group Publishing Limited.

Henseler, J.; Ringle, M. e Sarstedt, M. (2015). A new criterion for assessing discriminant validity in variance-based structural equation modeling. Journal of the Academy of Marketing Science, 43 (1), 115-135.

Marôco, J. (2010). Análise de equações estruturais. fundamentos teóricos, software e aplicações. Lisboa: Report Number.

Mitchell, R., Agle, B. \& Wood, D. (1997). Toward a theory of stakeholder identification and salience: defining the principle of who and what really counts. Academy of Management Review, 22(4), 853-886.

Moreno, M.; Molina, C. \& Moreno, R. (2014). Tourist attractions as a moderating element in explanatory models for loyalty development. Tourism and Management Studies, 10 (1),112-118.

Nunnally, J. (1978). Psychometric theory. New York: MacGraw-Hill.

Ringle, C.; Wende, S. \& Will, A. (2005). SmartPLS 2.0.M3. Hamburg: SmartPLS. Consultado em junho, 22, $2015 \mathrm{em}$ http://www.smartpls.com.

Ringle, C.; Wende, S. \& Becker, J-M. (2015a). SmartPLS 3. Hamburg: SmartPLS. Consultado em junho, 22, $2015 \mathrm{em}$ http://www.smartpls.com.

Ringle, C.; Wende, S. \& Becker, J-M. (2015b). SmartPLS 3. Hamburg: SmartPLS. Consultado em junho, 22, $2015 \mathrm{em}$ http://www.smartpls.de/documentation/srmr

Roldán, J. \& Sánchez-Franco, M. (2012). Variance-based structural equation modeling: guidelines for using partial least squares in information systems research. In M. Mora, O. Gelman, A. Steenkamp \& M. Raisinghani (eds.), Research methodologies, innovations and philosophies in software systems engineering and information systems (pp. 193-221). Hershey, PA: IGI Global.

Sarstedt, M.; Ringle, C.; Henseler, J. \& Hair, J. (2014). On the Emancipation of PLS-SEM: A Commentary on Rigdon (2012). Long Range Planning, 47, 154-160.

Sharma, S. \& Henriques, I. (2005): Stakeholder influences on sustainability practices in the Canadian forest products industry. Strategic Management Journal, 26(2), 159-180.

Sharma, S. \& Starik, M. (2004). Stakeholders, environment and society: multiple perspectives, emerging consensus. In Sharma \&, Starik (eds.). New perspectives on research in corporate sustainability: stakeholders, environment and society (pp.1-22). Northampton: EEA Publishing.

Tenenhaus, M.; Vinzi, V.; Chatellin, J. \& Lauro, C. (2005). PLS path modeling. Computational Statistics and Data Analysis, 28(1), 159205.

Vieira, A. (2009). ABC do LISREL interactivo - Um exemplo prático de Modelação em Equações Estruturais. Lisboa: Sílabo.
Submetido: 12.05 .2014

Aceite: 25.11 .2014 